\title{
Fertilización Foliar de Fósforo con sus diferentes dosis; para el rendimiento del cultivo de frijol castilla (Vigna unguiculata L.) en la provincia de Barranca
}

\section{Foliar fertilization of phosphorus with it's different doses; for the performance of the cultivation of frijol castilla (Vigna unguiculata L.) in the province of Barranca}

\author{
Dante Daniel Cruz Nieto ${ }^{1}$, Luis Alfredo Arias Rosales ${ }^{2}$, Francisco Espinoza \\ Montesinos $^{2}$, José del Carmen Ramírez Maldonado², Juan Jorge Maguiña Aguirre ${ }^{3}$ y \\ Edgar Amador Espinoza Montesinos ${ }^{4}$
}

\section{RESUMEN}

Esta investigación está basado en la fertilización de fósforo en el cultivo de frijol castilla en la Provincia de Barranca, se determinó la dosis adecuada para obtener mayor rendimiento. Para esto se realizó el experimento en el Fundo los Anitos, esto se aplicó en tres momentos y sus tratamientos son: $\mathrm{T}_{1}=0, \mathrm{~T}_{2}=0,5, \mathrm{~T}_{3}=1,0 \mathrm{y} \mathrm{T}_{4}=1,5$ litros por 200 l. de agua. Se empleó el diseño de bloque completamente al azar, la operación estadística que se utilizó fue el análisis de varianza y la Prueba de Duncan. Obtenidos los resultados de las evaluaciones, se determinó que el $\mathrm{T}_{4}$ alcanzó el mayor rendimiento con 2,9 Tm/ha., las demás evaluaciones en campo obtuvieron buenos resultados con mayor dosis estos son: la altura de planta, número de flores por planta, muestreo de vainas y peso por planta, siendo todos estadísticamente homogéneos. En cuanto a los datos obtenidos en laboratorio como longitud de vaina, número de granos, peso de 100 semillas, no mostraron diferencias significativas, los datos de Análisis foliar sobresalió el $\mathrm{T}_{4}$ con $0,72 \mathrm{~g}$ de fósforo/100 de materia seca y costo beneficio $\mathrm{S} /$ 0,53 Nuevos Soles.

Palabras claves: frijol castilla; fósforo; rendimiento y rentabilidad.

\footnotetext{
Universidad Nacional José Faustino Sánchez Carrión. Huacho, Perú.

2 Universidad Nacional Santiago Antúnez de Mayolo. Huaraz, Perú.

3 Colegio 21571 Ricardo Palma Soriano-Hacienda Araya Grande. Barranca, Perú.

4 Universidad para el Desarrollo Andino. Lircay - Huancavelica, Perú.

(C) Los autores. Este artículo es publicado por la Revista Aporte Santiaguino de la Universidad Nacional Santiago Antúnez de Mayolo. Este es un artículo de acceso abierto, distribuido bajo los términos de la Licencia Creative Commons Atribución-NoComercial-CompartirIgual 4.0 Internacional. (http://creativecommons.org/licenses/ by-nc-sa/4.0/), que permite el uso no comercial, distribución y reproducción en cualquier medio, siempre que la obra original sea debidamente citada.
} 


\begin{abstract}
This research is based on the fertilization of phosphorus in the cultivation of Castilian beans in the Province of Barranca, the appropriate dose was determined to obtain a higher yield. For this the experiment was carried out in the Fundo Los Anitos, this was applied in three moments and its treatments are: $\mathrm{T} 1=0, \mathrm{~T} 2=0.5, \mathrm{~T} 3=1,0$ and $\mathrm{T} 4$ $=1,5$ liters per 2001 . of water. The block design was used completely at random, the statistical operation that was used was the analysis of variance and the Duncan test. Obtained the results of the evaluations, it was determined that the T4 reached the highest yield with 2,9 Tm / ha., The other evaluations in the field obtained good results with higher doses, these are: plant height, number of flowers per plant, sampling of pods and weight per plant, all being statistically homogeneous. Regarding the data obtained in the laboratory as length of pod, number of grains, weight of 100 seeds, did not show significant differences, the data of leaf analysis stood out the T4 with 0,72 $\mathrm{g}$ of phosphorus/100 of dry matter and cost benefit S/ 0,53 Nuevo Soles.
\end{abstract}

Keywords: castilla bean; phosphorus; yield and profitability.

\title{
INTRODUCCIÓN
}

El frijol castilla conocido como frijol caupí, es una leguminosa de mayor consumo a nivel mundial, que se ha cultivado desde el periodo neolítico pues se remonta desde hace 5 a 6 mil años y que tiene su origen en el conteniente africano e India según Phansak et al. (2005). Asimismo su valor nutricional es muy favorable, por esta razón se viene sembrando en todo el mundo.

En nuestro país dicho cultivo se viene produciendo en Piura, Loreto, Lambayeque, Ucayali, Lima e Ica; ya sea por sus condiciones edafoclimática que son óptimas para el desarrollo favorable de este cultivo. Sin embargo esta necesidad de obtener mayor producción favorece a los agricultores a sembrar más áreas para el consumo del mercado nacional e internacional.

Cabe mencionar que nuestra Provincia de Barranca no es ajeno a la producción de esta leguminosa, por lo que estos últimos años hasta la fecha se viene cultivando más áreas de siembra para fines de comercialización en el mercado nacional e internacional siendo Estados Unido, Europa y China sus principales compradores.

Por otra parte, Quisphe (2010), determina en su trabajo de investigación acerca de los tratamientos $\mathrm{T}_{1}$ : Bayfolan en dosis de $2 \mathrm{cc} / 1$., $\mathrm{T}_{2}$ : Nitrofoska en dosis de $2 \mathrm{gr} / 1, \mathrm{~T}_{3}$ : Millerplex en dosis de 2g/l., $\mathrm{T}_{4}$ : Algreen 2cc/1. y $\mathrm{T}_{0}$ : Sin foliar. Obteniéndose buen resultado el $\mathrm{T}_{2}$ con $2,31 \mathrm{~kg}$, en comparación a los demás. Por lo que se concluye que el producto que tiene mayor concentración de fósforo (Nitrofoska) es adecuado.

Barbagelata (2012), investigó acerca de aplicación de fósforo en soja, con tratamientos: $\mathrm{T}_{1}$ con $0, \mathrm{~T}_{2}$ (Dualfos) con $50 \mathrm{l}$. / ha, $\mathrm{T}_{3}$ (Dualfos) con $100 \mathrm{l}$. / ha, $\mathrm{T}_{4}$ (Dualfos) con 
50+50 l/ ha, $\mathrm{T}_{5}(\mathrm{SFT}) \operatorname{con} 27,5 \mathrm{~kg} / \mathrm{ha}, \mathrm{T}_{6}(\mathrm{SFT}) \operatorname{con} 55 \mathrm{~kg} / \mathrm{ha}, \mathrm{T}_{7}$ (FMA) con $50 \mathrm{~kg} /$ ha, $\mathrm{T}_{8}$ (SFT) con $100 \mathrm{~kg} / \mathrm{ha}$. Obteniéndose mayor rendimiento en $\mathrm{T}_{8}$ con $3583 \mathrm{~kg} / \mathrm{ha}$, sin embargo las aplicación liquido alcanzó buena respuesta en $\mathrm{T}_{4}$ con $3543 \mathrm{~kg} / \mathrm{ha}$. Lo que determina que la dosis líquido de fósforo obtuvo buen resultado.

Con respecto a las funciones del fósforo; FAO (2002), expone que el fósforo suple de 0,1 a 0,4 por ciento del extracto seco de la planta, es importante en transferencia de energía. Por eso es esencial para la fotosíntesis y en su fisiología e indispensables para el desarrollo de los tejidos. Es deficiente en mayoría de suelos agrícolas dónde la fijación limita su disponibilidad.

CIA/UCR (2002), afirma que el fósforo es constituyente del ATP, ácidos nucleicos, fosfolípidos y ciertas enzimas. Cumple una función importante en el sistema de transferencia de energía dentro de la planta. El P es esencial para el crecimiento radical, en el proceso de floración, y en la formación de frutas y semillas. El P se aplica usualmente al suelo en cultivos frutícolas y pocas veces se realizan aspersiones foliares, quizás en parte debido a su lenta absorción foliar de la mayoría de las fuentes, especialmente las sales como los fosfatos de amonio.

Romheld y Fouly (1999), manifiestan que es necesario conocer la etapa de absorción de nutrientes para que la fertilización foliar sea efectiva. Se debe tener en cuenta que es específica en cultivo, la época de aplicación y el sitio de la aplicación. Como ejemplo la absorción de fósforo (P) es regulada por el estado nutricional de la planta, es decir, la planta absorbe más nutriente si éste se encuentra en deficiencia.

En cuanto a la utilización del producto Folix Phos; FARMAGRO (2017), informa que Folix Phos es un nutriente órgano mineral con alto contenido de Fósforo, 100\% activado orgánicamente por ácidos fúlvicos, debido a su avanzada y compleja formulación permite además el aporte de aminoácidos libres lo cual facilita la asimilación de nutrientes. Tambien participa activamente en los procesos de enraizamiento, desarrollo, crecimiento y multiplicación; aplicado en forma foliar en los momentos críticos, aporta una excelente y adecuada nutrición de acuerdo al estado fenológico.

Por tal motivo el objetivo de esta investigación fue evaluar el efecto de las diferentes dosis de fertilización foliar de fósforo en el rendimiento del cultivo de frijol castilla (Vigna unguiculata L.), en la Provincia de Barranca, Región Lima, por lo que se ha realizado este experimento de aplicación de fósforo; ya que no hay una fertilización estable en cuanto a su dosis y momento de aplicación. Esta investigación consta de cuatro tratamientos $\mathrm{T}_{1}=0.0, \mathrm{~T}_{2}=0.5, \mathrm{~T}_{3}=1.0 \mathrm{y} \mathrm{T}_{4}=1.5$ litros por cilindro, $\mathrm{y}$ la estadística que se empleo fue el Diseño de Bloques Completamente al Azar con su Análisis de Varianza y para la comparación de resultados en su significancia se empleó la Prueba de Duncan al $95 \%$ de confiabilidad.

Es importante mencionar que el manejo agronómico como es el caso de la fertilización al suelo, control fitosanitario, fue homogéneo para todos los tratamientos solo se varió 
la dosis de aplicación foliar de fósforo; y las evaluaciones en el campo y laboratorio se hizo conforme a lo establecido en el proyecto de investigación.

Esta investigación propone a que cada vez más se realice parcelas demostrativas en cuanto a las diferentes dosis y momentos de aplicación de fósforo, con fines de obtener mayor rendimiento y calidad en la Provincia de Barranca.

\section{MATERIALES Y MÉTODOS}

Se realiza en el fundo los anitos ubicado en el Distrito de Barranca, Provincia de Barranca, Región Lima. Bajo las condiciones agrícolas de la zona.

Se trata de una investigación aplicada, porque los resultados nos permitirán hacer las recomendaciones sobre el uso de la fertilización foliar de fósforo, en el cultivo de frijol castilla. Pues con este experimento se obtendrá un diagnóstico favorable.

En cuanto al análisis estadístico utilizado en la investigación se empleó el análisis de varianza con la Prueba de Fisher al 5 \%; ya que según Arriaza (2006), una Prueba de homogeneidad en cada grupo puede ocurrir que las medias sean diferentes pero con el mismo grado de dispersión de los datos. (Tabla 1).

Tabla 1. Análisis de Varianza de bloques y tratamiento aleatorizados

\begin{tabular}{|c|c|c|c|c|c|c|}
\hline $\begin{array}{l}\text { Fuente de } \\
\text { Variación }\end{array}$ & $\mathrm{SC}$ & Gl & $\mathrm{CM}$ & $\begin{array}{l}\text { Modelo I } \\
\text { E(CM) }\end{array}$ & $\begin{array}{l}\text { Modelo II } \\
\text { E(CM) }\end{array}$ & F.cal \\
\hline Bloques & $\mathrm{SCb}$ & $b-1$ & $\mathrm{CM} \mathrm{b}=\frac{\mathrm{SCb}}{(\mathrm{b}-1)}$ & $\frac{\sigma_{\mathrm{e}}^{2}+\sum \beta \mathrm{t}_{\mathrm{j}}^{2}}{(\mathrm{~b}-1)}$ & $\sigma_{\mathrm{e}}^{2}+t \sigma_{\beta}^{2}$ & $\frac{\mathrm{CM}_{\mathrm{b}}}{\mathrm{CM}_{\mathrm{e}}}$ \\
\hline Tratamiento & SCtr & $\mathrm{T}-1$ & $C M_{t r}=\frac{S C_{t r}}{(t-1)}$ & $\frac{\sigma_{e}^{2}+\sum t_{j}^{2}}{(t-1)}$ & $\sigma_{\mathrm{e}}^{2}+\underset{\mathrm{t}}{\mathrm{b} \sigma^{2}}$ & $\frac{\mathrm{CM}_{\mathrm{tr}}}{\mathrm{CM}_{\mathrm{e}}}$ \\
\hline Error & $\mathrm{SCe}$ & $(\mathrm{b}-1)(\mathrm{t}-1)$ & $C M_{e}=\frac{S_{e}}{(b-1)(t-1)}$ & $\sigma_{\mathrm{e}}^{2}$ & $\sigma_{\mathrm{e}}^{2}$ & \\
\hline Total & SCt & $b t-1$ & & & & \\
\hline
\end{tabular}

Fuente: Núñez y Tusell (2007), "Regresión y Análisis de Varianza” España

\section{Prueba de Duncan}

López y González (2014), manifiesta que es un procedimiento usado ampliamente para comparar todas las parejas de medias es el de la prueba de intervalos múltiples desarrollada por Duncan o como también comparación de las medias de tratamientos todos contra todos. 


\section{Tratamientos de estudio}

Esta investigación consta de la aplicación del foliar fosforo (Folix Phos) en tres momentos: a los 8 días (primera semana), 15 días (segunda semana) y a los 40 días después de la siembra. En la tabla 2, se muestra las dosis de aplicación del fosforo en líquido.

Tabla 2. Aplicación de fosforo (Folix Phos), según los tratamientos

\begin{tabular}{|c|c|c|c|c|c|c|}
\hline \multirow{3}{*}{ Tratamiento } & \multicolumn{5}{|c|}{ Aplicación del foliar fósforo } & \multirow{3}{*}{$\begin{array}{l}\text { Total/ } \\
\text { hectárea }\end{array}$} \\
\hline & \multirow{2}{*}{$\begin{array}{c}1 / 200 \\
1\end{array}$} & \multirow{2}{*}{$\begin{array}{c}\text { Parcela } \\
(\mathrm{ml} / 0.3 \mathrm{l})\end{array}$} & \multicolumn{3}{|c|}{ Momentos (1./ha) } & \\
\hline & & & $1 \mathrm{er}$ & $2 \mathrm{do}$ & 3er & \\
\hline $\mathbf{T}_{1}$ & 0,0 & 0,0 & 0,0 & 0,0 & 0,0 & 0 \\
\hline \multirow{4}{*}{$\mathbf{T}_{2}$} & \multirow{4}{*}{0,5} & \multirow{4}{*}{0,75} & & & 0,5 & \\
\hline & & & & & 0,5 & \\
\hline & & & & & 0,75 & \\
\hline & & & & & 1,75 & \\
\hline $\mathbf{T}_{3}$ & 1,0 & 1,5 & 1 & 1 & 1,5 & 3,50 \\
\hline $\mathbf{T}_{4}$ & 1,5 & 2,25 & 1,5 & 1,5 & 2,25 & 5,25 \\
\hline
\end{tabular}

Nota: Cabe mencionar que solo se varió en la dosis de aplicación del foliar fosforo (Folix Phos) en cada parcela. Las demás labores agronómicas fueron igual para todo el experimento.

\section{RESULTADOS Y DISCUSIÓN}

\section{Altura de planta de frijol castilla}

De acuerdo con el análisis estadístico se determinó los resultados de la Prueba de Duncan en la tabla 3, que detalla los resultados son estadísticamente homogéneos por lo que todos son de un mismo grupos (a), lo cual significa que no hay diferenciación de los resultados en cuanto altura de planta. 
||Dante Cruz, Luis Arias, Francisco Espinoza, José del Carmen Ramírez, Juan Maguiña y Edgar Espinoza

Tabla 3. Prueba de Duncan de altura de planta

\begin{tabular}{cccc}
\hline \multirow{2}{*}{ Tratamiento } & Dosis de fósforo (Folix Phos) & $\begin{array}{c}\text { Altura } \\
(\mathrm{cm})\end{array}$ & $\begin{array}{c}\text { Duncan } \\
\text { Agrupamiento }\end{array}$ \\
\hline $\mathrm{T}_{4}$ & $1,5 \mathrm{~L} / 200 \mathrm{~L}$ & 49,83 & $\mathbf{a}$ \\
& $1,0 \mathrm{~L} / 200 \mathrm{~L}$ & 48,95 & $\mathbf{a}$ \\
$\mathrm{T}_{3}$ & $0,5 \mathrm{~L} / 200 \mathrm{~L}$ & $\mathbf{a}$ \\
$\mathrm{T}_{2}$ & $0,0 \mathrm{~L} / 200 \mathrm{~L}$ & 48,06 & $\mathbf{a}$ \\
$\mathrm{T}_{1}$ & & $\mathbf{a}$ \\
\hline
\end{tabular}

Nota: Promedios con la misma letra son homogéneos

De la misma manera se observa el coeficiente de variación de $9,72 \%$ que significa que hay una variación de los promedios de altura de planta tomados en campo. También se explica el figura 1, que hay un aumento de altura en cuanto a la aplicación de dosis de fósforo lo cual se determinó que la mayor altura es $\mathrm{T}_{4}$ con 49,83 cm. Esto con concuerda con Guevara (2012), que en la aplicación de fosforo (Strong-Phos) con dosis de $\mathrm{T}_{5}(1200 \mathrm{ml} / 200 \mathrm{l}$ ) alcanzó mayor rendimiento el sitio de aplicación para que la fertilización foliar sea efectiva.

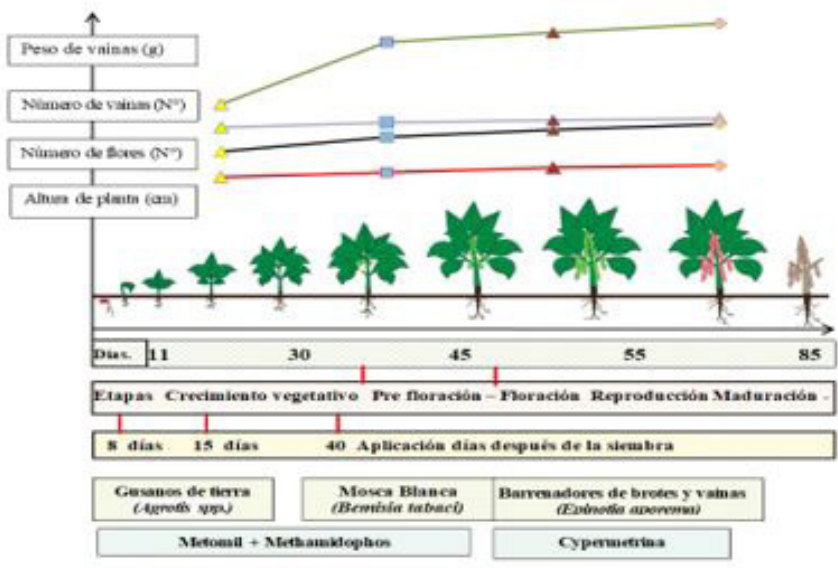

\begin{tabular}{|c|c|c|c|c|}
\hline & \multicolumn{4}{|c|}{ Tratamientes } \\
\hline Evaluaciones de muestreo & $T_{1} \square$ & $\mathbf{T}_{2} \square$ & $T_{3} \square$ & $\mathbf{T}_{4} \mathrm{D}$ \\
\hline Peso de vainas por planta (muestreo) ( $(2)$ & 86.04 & 113.00 & 117.05 & 121.01 \\
\hline Número devainas / planta (muestreo) $\mathbb{N}^{\circ}$ & 35.63 & 41.72 & 44.77 & 47.43 \\
\hline Número de flores por planta (N) & 24,43 & 26.39 & 28.53 & 29.39 \\
\hline Altura de planta $(\mathrm{cm})$ & 45,91 & 48.07 & 48.95 & 49.83 \\
\hline
\end{tabular}

Figura 1. Efecto de dosis de fósforo, según las evaluaciones de muestreo 


\section{Número de flores por planta}

Según la tabla 4, determina que el $\mathrm{T}_{4}$ con 29,39 flores por planta obtuvo la mayor cantidad de flores en referencia a los demás, lo cual es similar al $\mathrm{T}_{3}, \mathrm{~T}_{2}$ en agrupación de (ab) y el $\mathrm{T}_{2}$ es similar al $\mathrm{T}_{1}$ en Grupo de (b), por tal motivo se afirma que las dosis de aplicación de fósforo tienen relación homogénea.

Tabla 4. Prueba de Duncan de número de flores

\begin{tabular}{ccccc}
\hline Tratamiento & Dosis de fósforo (Folix Phos) & $\begin{array}{c}\text { Número de } \\
\text { flores }\left(\mathrm{N}^{\circ}\right)\end{array}$ & $\begin{array}{c}\text { Duncan } \\
\text { Agrupamiento }\end{array}$ \\
\hline $\mathrm{T}_{4}$ & $1,5 \mathrm{~L} / 200 \mathrm{~L}$ & 29,39 & $\mathbf{a}$ & \\
& $1,0 \mathrm{~L} / 200 \mathrm{~L}$ & $\mathbf{a}$ & \\
$\mathrm{T}_{3}$ & $0,5 \mathrm{~L} / 200 \mathrm{~L}$ & 28,53 & $\mathbf{a}$ & $\mathbf{b}$ \\
& & 26,39 & $\mathbf{a}$ & $\mathbf{b}$ \\
$\mathrm{T}_{2}$ & $0,0 \mathrm{~L} / 200 \mathrm{~L}$ & & $\mathbf{b}$ \\
\hline
\end{tabular}

Nota: Promedios con la misma letra son homogéneos.

\section{Número de vainas por planta (Muestreo)}

Asimismo el coeficiente de variación es de 18,44 \%, indicar mediana variación entre promedios, también se detalla los resultados en la tabla 5 , que resalta mayor cantidad el $\mathrm{T}_{4}$ con 47,42 vainas por planta, además no hay variación en niveles, siendo todo estos resultados significativamente homogéneos (a).

Tabla 5. Prueba de Duncan de número de vaina por planta (muestreo)

\begin{tabular}{cccc}
\hline \multirow{2}{*}{ Tratamiento } & Dosis de fósforo (Folix Phos) & Vainas & $\begin{array}{c}\text { Duncan } \\
\left(N^{\circ}\right)\end{array}$ \\
\hline$T_{4}$ & $1,5 \mathrm{~L} / 200 \mathrm{~L}$ & 47,42 & $\mathbf{a}$ \\
& & & $\mathbf{a}$ \\
$\mathrm{T}_{3}$ & $1,0 \mathrm{~L} / 200 \mathrm{~L}$ & 44,77 & $\mathbf{a}$ \\
& & & $\mathbf{a}$ \\
$\mathrm{T}_{2}$ & $0,5 \mathrm{~L} / 200 \mathrm{~L}$ & 41,72 & $\mathbf{a}$ \\
& & & $\mathbf{a}$ \\
$\mathrm{T}_{1}$ & $0,0 \mathrm{~L} / 200 \mathrm{~L}$ & 35,62 & $\mathbf{a}$ \\
\hline
\end{tabular}

Nota: Promedios con la misma letra son homogéneos. 


\section{Peso de vainas por planta (Muestreo)}

Seguido del análisis anterior se precisó los datos obtenidos de la Prueba de Duncan en la tabla 6 , señala que el $\mathrm{T}_{4}$ con $121,01 \mathrm{~g}$, tiene buen peso de vainas por planta y es estadísticamente homogéneo a los demás tratamientos.

Tabla 6. Prueba de Duncan de peso de vainas por planta (muestreo)

\begin{tabular}{cccc}
\hline \multirow{2}{*}{ Tratamiento } & Dosis de fósforo (Folix Phos) & Peso (g.) & $\begin{array}{c}\text { Duncan } \\
\text { Agrupamiento }\end{array}$ \\
\hline $\mathrm{T}_{4}$ & $1,5 \mathrm{~L} / 200 \mathrm{~L}$ & 121,01 & $\mathbf{a}$ \\
& & & $\mathbf{a}$ \\
$\mathrm{T}_{3}$ & $1,0 \mathrm{~L} / 200 \mathrm{~L}$ & 117,05 & $\mathbf{a}$ \\
& & & $\mathbf{a}$ \\
$\mathrm{T}_{2}$ & $0,5 \mathrm{~L} / 200 \mathrm{~L}$ & 113,00 & $\mathbf{a}$ \\
& & & $\mathbf{a}$ \\
$\mathrm{T}_{1}$ & $0,0 \mathrm{~L} / 200 \mathrm{~L}$ & 86,04 & $\mathbf{a}$ \\
\hline
\end{tabular}

Nota: Promedios con la misma letra son homogéneos

\section{Rendimiento comercial (Tm. /ha)}

Por lo que corresponde a la Prueba de Duncan en la tabla 7, precisa los resultados estadísticos que son homogéneos, es decir no hubo variación en el agrupamiento Duncan siendo todos (a), es decir estadísticamente similares entre tratamientos, por lo tanto no influyó el efecto de dosis de fósforo (Folix Phos).

Tabla 7. Prueba de Duncan de rendimiento comercial

\begin{tabular}{cccc}
\hline Tratamiento & Dosis de fósforo (Folix Phos) & $\begin{array}{c}\text { Rendimiento } \\
(\mathrm{Tm} / \mathrm{ha})\end{array}$ & $\begin{array}{c}\text { Duncan } \\
\text { Agrupamiento }\end{array}$ \\
\hline $\mathrm{T}_{4}$ & $1,5 \mathrm{~L} / 200 \mathrm{~L}$ & 2,900 & $\mathbf{a}$ \\
& $1,0 \mathrm{~L} / 200 \mathrm{~L}$ & $\mathbf{a}$ & $\mathbf{a}$ \\
$\mathrm{T}_{3}$ & $0,5 \mathrm{~L} / 200 \mathrm{~L}$ & 2,732 & $\mathbf{a}$ \\
& & 2,584 & $\mathbf{a}$ \\
$\mathrm{T}_{2}$ & $0,0 \mathrm{~L} / 200 \mathrm{~L}$ & & $\mathbf{a}$ \\
$\mathrm{T}_{1}$ & 2,251 & $\mathbf{a}$ \\
\hline
\end{tabular}

Nota: Promedios con la misma letra son homogéneos 


\section{Longitud de vaina por tratamiento}

Su coeficiente de variación es de 5,75 \% que significa que son moderadamente variables. Según la Prueba de Duncan en la tabla 8 , se observa que todo el agrupamiento de Duncan son de una misma letra (a), siendo todas las parcelas estadísticamente homogéneos, es decir no hay variación de los resultados, en cuanto a la aplicación de fosforo.

Tabla 8. Prueba de Duncan de longitud de vaina por tratamiento

\begin{tabular}{cccc}
\hline Tratamiento & Dosis de fósforo (Folix Phos) & $\begin{array}{c}\text { Longitud } \\
(\mathrm{cm} .)\end{array}$ & $\begin{array}{c}\text { Duncan } \\
\text { Agrupamiento }\end{array}$ \\
\hline $\mathrm{T}_{4}$ & $1,5 \mathrm{~L} / 200 \mathrm{~L}$ & 16,86 & $\mathbf{a}$ \\
& & $\mathbf{a}$ \\
$\mathrm{T}_{3}$ & $1,0 \mathrm{~L} / 200 \mathrm{~L}$ & 16,52 & $\mathbf{a}$ \\
& & & $\mathbf{a}$ \\
$\mathrm{T}_{2}$ & $0,5 \mathrm{~L} / 200 \mathrm{~L}$ & 16,21 & $\mathbf{a}$ \\
$\mathrm{T}_{1}$ & $0,0 \mathrm{~L} / 200 \mathrm{~L}$ & & $\mathbf{a}$ \\
\hline
\end{tabular}

Nota: Promedios con la misma letra son homogéneos

\section{Número de granos por vaina}

Después del análisis anterior, se muestra la Prueba de Duncan en la tabla 9, que indica los tratamientos son estadísticamente homogéneos, es decir que los promedios son similares en cuanto a su agrupación de un mismo nivel (a), esto significa que no influenció las dosis.

Tabla 9. Prueba de Duncan de número de granos por vaina

\begin{tabular}{cccc}
\hline Tratamiento & Dosis de fósforo (Folix Phos) & N granos & $\begin{array}{c}\text { Duncan } \\
\text { Agrupamiento }\end{array}$ \\
\hline $\mathrm{T}_{4}$ & $1,5 \mathrm{~L} / 200 \mathrm{~L}$ & 12,06 & $\mathbf{a}$ \\
& $1,0 \mathrm{~L} / 200 \mathrm{~L}$ & & $\mathbf{a}$ \\
$\mathrm{T}_{3}$ & $0,5 \mathrm{~L} / 200 \mathrm{~L}$ & 11,80 & $\mathbf{a}$ \\
$\mathrm{T}_{2}$ & & 11,45 & $\mathbf{a}$ \\
& $0,0 \mathrm{~L} / 200 \mathrm{~L}$ & $\mathbf{a}$ & $\mathbf{a}$ \\
$\mathrm{T}_{1}$ & & 11,09 & $\mathbf{a}$ \\
\hline
\end{tabular}

Nota: Promedios con la misma letra son homogéneos 


\section{Análisis foliar (100 $\mathrm{g}$ de materia seca)}

Para el análisis de foliar se llevó muestra de hojas de frijol castilla al Instituto de Innovación Agraria - Huaral, y se determinó las concentraciones de fósforo en 100 gramos de materia seca. Los resultados, del cuadro 10 , muestran que el $\mathrm{T}_{4}$ es de 0,72 g en $100 \mathrm{~g}$ de materia seca, siendo el más alto con relación a las demás dosis del ' $\mathrm{T}_{3}$, $\mathrm{T}_{2} \mathrm{y} \mathrm{T}_{1}$. Además se puede notar que el $\mathrm{T}_{3}$ tiene la cantidad de fósforo normal con 0,59 g en $100 \mathrm{~g}$ de materia, por lo que significa que dicha concentración es adecuado en cuanto a lo retenido de fósforo en las hojas en condiciones normales de absorción (tabla 10).

Tabla 10. Análisis foliar de fósforo (g. /100g de materia seca) por tratamiento.

\begin{tabular}{ccccc}
\hline Tratamiento & Dosis & Resultados & Calificación & Valores Normales \\
& $1 /$ ha & $(\%)$ & Bajo & $0,30-0,70$ \\
\hline $\mathrm{T}_{1}$ & 0,0 & 0,22 & Bajo & $0,30-0,70$ \\
$\mathrm{~T}_{2}$ & 0,5 & 0,29 & Normal & $0,30-0,70$ \\
$\mathrm{~T}_{3}$ & 1,0 & 0,59 & Alto & $0,30-0,70$ \\
$\mathrm{~T}_{4}$ & 1,5 & 0,72 & & \\
\hline
\end{tabular}

Fuente: INIA (2016) “Análisis de fósforo en hojas". Laboratorio de suelos

\section{Análisis económico}

Con el fin de precisar la ganancia y gasto de cada tratamiento, se efectuó el análisis económico, esto se muestra en el cuadro 20 , lo cual indica que la mayor utilidad se obtuvo en le $\mathrm{T}_{4}$ con S/. 2797,39, seguido del $\mathrm{T}_{3}$ con S/. 2394,16, $\mathrm{T}_{2}$ con S/. 2046,92 y $\mathrm{T}_{1}$ con $\mathrm{S} / 1181,69$ estableciéndose que a esta dosis de fósforo (Folix Phos), se obtuvo mayor ganancia económica (tabla 11).

Tabla 11. Análisis económicos de los tratamientos

\begin{tabular}{cccccc}
\hline Tratamiento & $\begin{array}{c}\text { Costo prod. } \\
(\mathrm{S} /)\end{array}$ & $\begin{array}{c}\text { Rendto } \\
(\mathrm{Kg} / \mathrm{ha})\end{array}$ & $\begin{array}{c}\text { Utilidad } \\
(\mathrm{S} /)\end{array}$ & $\begin{array}{c}\text { Ganancia } \\
1 \mathrm{~S} /\end{array}$ & $\begin{array}{c}\text { Costo - beneficio } \\
(\mathrm{S} /)\end{array}$ \\
\hline $\mathrm{T}_{1}$ & 5121,11 & 2251 & 1181,69 & 1 & 0,23 \\
$\mathrm{~T}_{2}$ & 5188,28 & 2584 & 2046,92 & 1 & 0,39 \\
$\mathrm{~T}_{3}$ & 5255,44 & 2732 & 2394,16 & 1 & 0,46 \\
$\mathrm{~T}_{4}$ & 5322,61 & 2900 & 2797,39 & 1 & 0,53 \\
\hline
\end{tabular}




\section{CONCLUSIONES}

Se determinó que el mayor rendimiento comercial de frijol castilla, se obtuvo aplicando la dosis de 1.5 litros de fósforo (Folix Phos) por 200 l., alcanzando $2900 \mathrm{Kg}$ por hectárea, siendo mayor en comparación al $\mathrm{T}_{1}=0,0$ litros / ha (testigo) con $2251 \mathrm{Kg} /$ ha.

En las evaluaciones de la altura de planta, cantidad de flores, número de vainas y peso de vainas fue mayor con la aplicación de 1,5 litros de fósforo (Folix Phos) por 2001.

En cuanto a las evaluaciones de laboratorio como longitud de vaina, número de granos, peso de 100 semillas y análisis de fósforo (g. /100 g de materia seca), sobresalió $\mathrm{T}_{4}$

El mejor resultado en el análisis foliar de fósforo, se obtuvo con la dosis de 1.5 1. / ha, siendo de 0,72 g. /100 g materia seca; evidenciando la influencia favorable.

Se determinó que el mayor costo/ beneficio se obtuvo con la dosis $\mathrm{T}_{4}$ con $\mathrm{S} /$. 0,53

Nuevo Soles, es decir que por cada sol invertido se gana más de la mitad, siendo más rentable en referencia a las demás dosis.

\section{RECOMENDACIONES}

Se debe realizar investigaciones acerca de otras dosis de fósforo, su época de aplicación del frijol castilla y en otras leguminosas y otros cultivos, con el fin de obtener mayor rendimiento y calidad de frutos.

Es recomendable tomar muestras de suelo de forma escalonada o en diagonal de ambos lados del terreno y llevar la muestra representativa de un $1 \mathrm{~kg}$ al análisis de suelo. Con la finalidad de obtener las dosis recomendables de Macronutrientes y de este modo evitar la pérdida de nutrientes.

Programar y realizar las labores: culturales, fitosanitarias y de fertilización; con el fin de prevenir y controlar problemas de plagas y enfermedades que afecten directamente al cultivo del frijol castilla.

Incorporar dentro del programa de fertilización de frijol castilla y otros cultivos, ya que la utilización de fertilizante foliar de fósforo con la dosis de aplicación de 1.5 1. / 200 l; obtuvo buenos resultados en su rendimiento y calidad de vaina.

\section{REFERENCIAS BIBLIOGRÁFICAS}

Arriaza, M. 2006. "Guía práctica de análisis de datos". Instituto de investigación y Formación Agraria y Pesquera- IFAPA. Libro - Depósito Legal: I.S.B.N.: 84611-1661-5. Córdova - España. 7.

Barbagelata, P. 2012. "Evaluación de Fertilizantes Fosfatados en Soja campaña 2011/12". INTA (Instituto Nacional de Tecnología Agropecuaria) - EEA Paraná - Argentina. Artículo científico. 4 y 5.

CIA/UCR. 2002. "Laboratorio de suelos y foliares", Centro de Investigaciones Agronómicas y Universalidad de Costa Rica. Edición Meléndez y Molina y Conferencista Barquero, G. y otros. Costa Rica. 83.

FAO. 2002. "Los fertilizantes y su uso”, está basada de la información de. "World Fer- 
tilizer use Manual, FAO (Organización de las Naciones Unidas para la Alimentación y la Agricultura) 1992, IFA, París”, Página Web <http://www.fertilizer. org $>$ [Consulta: 06-07-2017].

FARMAGRO. 2017. "Ficha técnica Folix Phos" Perú. Página web <http://www.farmagro.com.pe/media_farmagro/uploads/ficha_tecnica/folix_phos_ficha_tecnica.pdf> [Consulta: 06-12-2017].

Guevara, J. 2012. "Efecto de cinco dosis de bionutriente líquido (Strong-Phos) en el rendimiento del cultivo de caupí (Vigna unguiculata (L.) Walp), en el Fundo Miraflores - UNSM-T”. Para optar el Título Profesional de Ingeniero Agrónomo. Universidad Nacional de San Martín - Tarapoto. Tarapoto - Perú. Página 54 y 55.

INIA. 2016. "Análisis de fósforo en hojas", Instituto Nacional de Innovación Agraria - Estación Experimental Agraria Donoso Kiyotada Miyagawa. -Huaral - Perú

López, E. y González, B. 2014. "Diseño y Análisis de Experimentos Fundamentos y Aplicaciones en Agronomía”, 2a . Edición Revisada y Ampliada. GUATEMALA. Página 52.

Núñez, V. y Tusell, F. 2007. “Regresión y Análisis de Varianza”. España. Página 143 y 176.

Phansak, P.; Taylor P. y Mongkolporn, O. 2005. "Genetic diversity in yardlong bean (Vigna unguiculata ssp. sesquipedalis) and related Vigna species using sequence tagged microsatellite site analysis". Scientia Horticulture 106 (1):137- 146.

Quisphe, M. 2010. “Determinación de la influencia de fertilización foliar como complemento a la fertilización edafológica en la producción de fréjol arbustivo variedad INIAP - 414 Yunguilla en el Cantón Paute" Tesis previa a la obtención del Título de Ingeniero Agropecuario Industrial. Universidad Politécnica Salesiana. Azuay - Ecuador. 48 y 88.

Romheld, V. y Fouly, M. 1999. “Aplicación Foliar de Nutrientes: Retos y Limites en la Producción Agrícola. Informaciones Agronómicas No. 48. Fertilizer Society of Thailand. Bangkok, Thailand. 11-14.

Recepción: 17/11/2018

Aceptación: 20/12/2018

\section{Correspondencia}

Dante Daniel Cruz Nieto

daniel2262@hotmail.com 